\title{
Vestibular Test Results of a Patient with Cerebral Microangiopathy and Idiopathic Thrombocytopenia
}

\author{
Bre Myers ${ }^{1}$ Deirdre Creegan ${ }^{1}$ Elisa Hoyos ${ }^{1}$ \\ ${ }^{1}$ Osborne College of Audiology, Salus University, Elkins Park, \\ Pennsylvania
}

Address for correspondence Bre Myers, AuD, PhD, bmyers@salus.edu.

J Am Acad Audiol 2020;31:620-626.

\begin{abstract}
Keywords

- cerebral microangiopathy

- computerized dynamic posturography

- idiopathic thrombocytopenia

- video head impulse test

- videonystagmography

- rotational chair

- sinusoidal harmonic acceleration

- bilateral hypofunction

Background Cerebral microangiopathy (CM) is a general term for small ischemic changes related to small blood vessels in the brain. Immune thrombocytopenia (ITP) is a rare acquired autoimmune disease that is characterized by low platelet count and increased risk of hemorrhage. We describe vestibular testing completed on a 64-yearold patient with CM and ITP presenting with complaints of general instability and listing while walking, decreased stability in dark environments, and blurred vision with head movement. Results indicated a severe loss of horizontal semicircular canal function bilaterally with a somatosensory-dependent pattern of postural instability.

Purpose This case emphasizes the importance of vestibular testing to determine the level of involvement of the vestibular system in the patient's symptomatology with known CM. Due to the increased risk of severe injury in those with ITP and the unknown effects this may have on the vestibular and balance systems, what is known about the pathophysiology of ITP and possible implications are reviewed.

Research Design Case report.

Data Collection and Analysis Previous audiological and medical records were obtained. Vestibular testing included videonystagmography; video head impulse test (VHIT); rotational chair including sinusoidal harmonic acceleration (SHA), step velocity, SHA with fixation, and visual vestibular SHA; computerized dynamic posturography battery including sensory organization test (SOT), motor control test, and adaptation test.

Results Audiological evaluation indicated a mild to moderately severe sensorineural hearing loss bilaterally. Ocular motor evaluation results indicated abnormal "cogwheel" like tracings in smooth pursuit, hypometric saccades with abnormally increased latencies and reduced optokinetic gain. The patient also demonstrated apogeotropic nystagmus during head/body right and head/body left positions when fixation was denied. Bilateral bithermal calorics indicated a bilateral hypofunction. Horizontal vHIT results indicated reduced gain bilaterally. SHA results confirmed significant gain reductions at all frequencies tested and step velocity results indicated reduced gain and reduced time constants during all prerotary and postrotary measures. SOT results indicated a somatosensory-dependent pattern (i.e., reduced equilibrium index scores and falls in conditions 4,5 , and 6).
\end{abstract}

received

July 24, 2019

accepted after revision

January 25, 2020

published online

April 27, 2020
Copyright $\odot 2020$ by the American

Academy of Audiology. All rights reserved. Thieme Medical Publishers,

Inc., 333 Seventh Avenue, New York,

NY 10001, USA.

Tel: +1(212) 760-0888.
DOI https://doi.org/ 10.1055/s-0040-1709443. ISSN 1050-0545. 
Conclusions These findings emphasize the importance of comprehensive vestibular evaluations of patients with CM, ITP, and complaints of instability. Identification and quantification of residual peripheral vestibular function as well as central system involvement can provide significant information pertinent to falls risk reduction and vestibular rehabilitation strategies, outcomes, and goals.

Cerebral microangiopathy $(\mathrm{CM})$ is characterized by changes in small brain vessels including small arteries, arterioles, capillaries, and small veins. ${ }^{1}$ It is primarily diagnosed through neuroimaging studies such as magnetic resonance imaging (MRI). Neurologic symptoms of CM include reports of seizures, vertigo, incontinence, and other stroke or transient ischemic attacks. Gait apraxia has also been reported in $27.8 \%$ of patients with $\mathrm{CM}^{2}$ Current treatment of $\mathrm{CM}$ is not clearly defined. While there is promising information regarding reducing risk of developing CM, there is currently no agreed upon therapeutic prevention or intervention strategy. ${ }^{3}$ Current classes of drugs used to prevent or treat $\mathrm{CM}$ include, but are not limited to, acetylcholinesterase inhibitors, anticoagulation medicines, anti-inflammatory agents, antiplatelet agents, and blood pressure lowering medicines. ${ }^{3}$

The overall prevalence of immune thrombocytopenia (ITP), also known as idiopathic thrombocytopenic purpura, is unknown, though a recent study estimates that it may be close to 23.6 per 100,000 adults. ${ }^{4}$ ITP is characterized by low platelet counts and is typically chronic in nature when diagnosed in adults. ${ }^{5}$ Chronic ITP is even less prevalent with estimates of 7.1 and 9.5 per 100,000 adults. ${ }^{4}$ Patients with chronic ITP can develop antibodies that target other tissues or organs in the body leading to the development of further illness and disease. Treatment strategies for ITP are dependent on the severity of the presentation. Often those with milder cases of ITP who have an acceptable platelet count are not actively treated rather they undergo routine monitoring. ${ }^{6}$ For those with lower platelet counts, corticosteroids are prescribed as a first course of action. Due to ITP's characteristic low platelet counts, patients with ITP are at risk for severe hemorrhage, due to decreased ability to clot. This increased risk of severe hemorrhage is an important consideration when evaluating a person's falls risk and general vestibular and balance function.

\section{Case Study}

\section{Vestibular/Balance History}

Our patient was a 64-year old male referred by his neurologist for vestibular evaluation due to progressively worsening balance and feeling like he was "veering to the right" while walking. The symptoms were first noticed 1 year prior to his date of evaluation, and had significantly progressed in the past 6 months. The patient reported that he felt the most unsteady when he walked in dark or dimly lit environments, particularly on uneven surfaces like his lawn. The patient reported that he had previously been seen by a physical therapist for vestibular rehabilitation, but had not perceived any benefit from the therapy. He has fallen and has had near falls on several occasions but had not yet sustained any serious injuries.

\section{Audiological Evaluation}

Comprehensive audiological testing performed at another facility 3 months prior revealed symmetric mild to moderately severe sensorineural hearing loss bilaterally. He reportedly had a significant history of work-related noise exposure. Speech recognition thresholds were found to be in agreement with pure-tone averages. Word recognition testing revealed a score of $88 \%$ at $65 \mathrm{~dB}$ HL in the right ear, and a score of $92 \%$ at $60 \mathrm{~dB}$ HL in the left ear. Tympanometry revealed normal middle ear function with ear canal volume, static compliance, and peak pressure to be within normal limits bilaterally.

\section{Medical History}

The following medical history was reported by the patient: arthritis, ITP, and high blood pressure. Current medications were listed as Cialis and Metoprolol. The patient denied any allergies, recreational drug use, tobacco use, or alcohol consumption. He reported a 30-year history of knee injuries including at a total knee replacement on the right side several years prior to the appointment. He reported that his initial diagnosis of ITP was made several years prior and was based in part due to low platelet levels. At the time of testing, he was under monitoring and not active treatment for ITP as his platelet levels were above the recommended cutoff. At the time of testing, he was under the care of his neurologist who had recently diagnosed him with CM based off of abnormal imaging studies. He denied any significant cognitive decline.

\section{Imaging Studies}

Results of MRI performed with and without contrast revealed mildly prominent ventricles and sulci, comparable to patient age. There was no reported evidence of mass or edema. Mild white matter signal abnormalities were documented and attributed to the patient's history of microangiopathy. No evidence of infarction or hemorrhage was reported. Cerebellar tonsils were noted to be in normal location and intracranial vascular flow voids were noted to be normal.

\section{Methods}

The following tests were performed at The Pennsylvania Ear Institute over the course of two appointments: Halmagyi Head Thrust; computerized dynamic posturography (CDP) battery including sensory organization test (SOT), motor control test 
(MCT), and adaptation test (ADT) using Neurocom's Equitest system; videonystagmography (VNG) battery including saccade, pursuit, optokinetic, gaze evoked/spontaneous nystagmus, Dix-Hallpike maneuvers, static positional testing, and bilateral bithermal air calorics; rotational chair studies including sinusoidal harmonic acceleration (SHA), step velocity (100 degrees/second clockwise and counterclockwise), visual fixation (VFX) and visual enhanced (VVOR) SHA testing via Micromedical VisualEyes system; and video head impulse test (vHIT) via Micromedical's Vorteq vHIT system.

\section{Results}

\section{Halmagyi Head Thrust}

Our patient was unable to maintain stable VFX and multiple corrective overt saccades were visualized during lateral head thrusts bidirectionally.

\section{Computerized Dynamic Posturography}

The CDP battery of tests was performed in the following order: SOT, MCT, and ADT. SOT measures are obtained by measuring (via a force plate) the amount of postural sway a person exhibits in a series of six different conditions. Each condition augments the ability to use either vision, somatosensory, or vestibular senses in a systematic manner. MCT evaluates motor control response of the lower extremities by quickly and sporadically moving the force plate forward and backward. ADT measures the ability to adapt to a sudden change including rotating toes up and rotating toes down.
SOT: Equilibrium index (EI) is calculated by measuring the amount of anterior and posterior sway in each condition. EI scores are compared with age-matched normative data for each condition as well as a composite EI. Normal EI scores were achieved during conditions 1,2 , and 3, with significantly reduced EIs and/or falls during conditions 4, 5, and 6, resulting in overall reduced composite EI ( - Fig. 1). MCT was completed next, and revealed that although the patient did fall into a normal range for his composite score, he had abnormally long latencies in both large forward and large backward translations (-Fig. 2). These measures were analyzed and verified to be an accurate representation of the patient's abilities. ADT was completed following MCT, and the patient was able to successfully adapt to both the toes up and toes down stimuli.

\section{Videonystagmography}

Ocular motor testing was performed using a 50-inch monitor and independent binocular video recording goggles. The patient was alert and compliant throughout the evaluation. No spontaneous or gaze-evoked nystagmus was observed with or without fixation. Smooth pursuit testing was within normal limits for gain and symmetry; however, small saccadic intrusions were visible on the eye tracing particularly at $0.4 \mathrm{~Hz}$ (-Fig. 3). Saccade testing indicated abnormal accuracy (repeated hypometric responses), prolonged latency, and reduced velocity measures (-Fig. 4). Optokinetic testing was performed at a rate 30 degrees/second and revealed reduced gain for left and right moving stimuli. Dix-Hallpike maneuvers were negative

\section{Sensory Organization Test (Sway Referenced Gain: 1.0) Equilibrium Score}
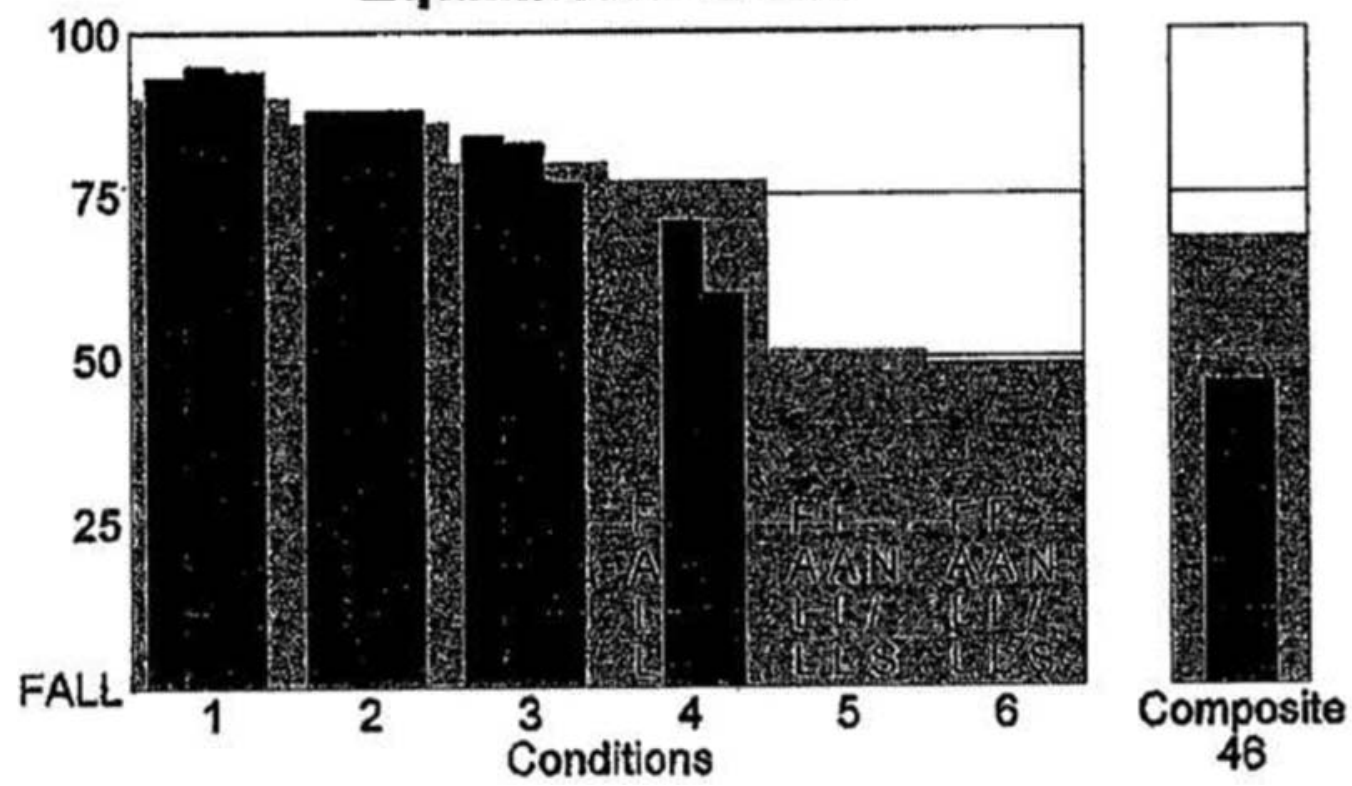

Fig. 1 Sensory organization test comprehensive test results depicting abnormal composite and individual equilibrium scores during conditions 4,5 , and 6 . Sensory analysis demonstrates ineffective use of vestibular and visual information resulting in a somatosensory-dependent configuration. Strategy analysis depicts abnormal ankle-dominant movements. Center of gravity alignment is normal. 


\section{Motor Control Test}
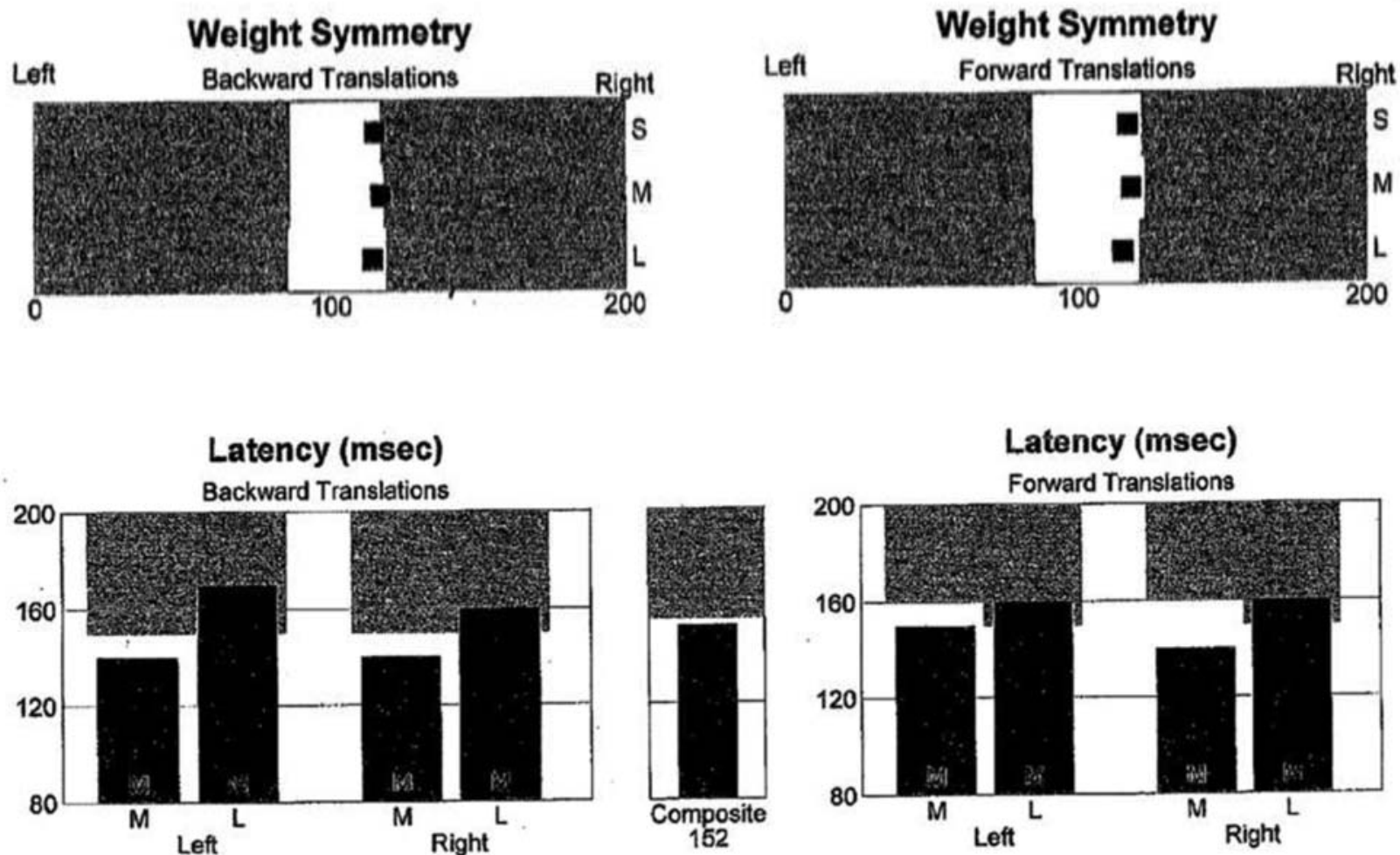

Fig. 2 Motor control test results indicating normal weight distribution during testing. Normal latencies were observed during all medium translations. Abnormally long latencies were observed during large translations.

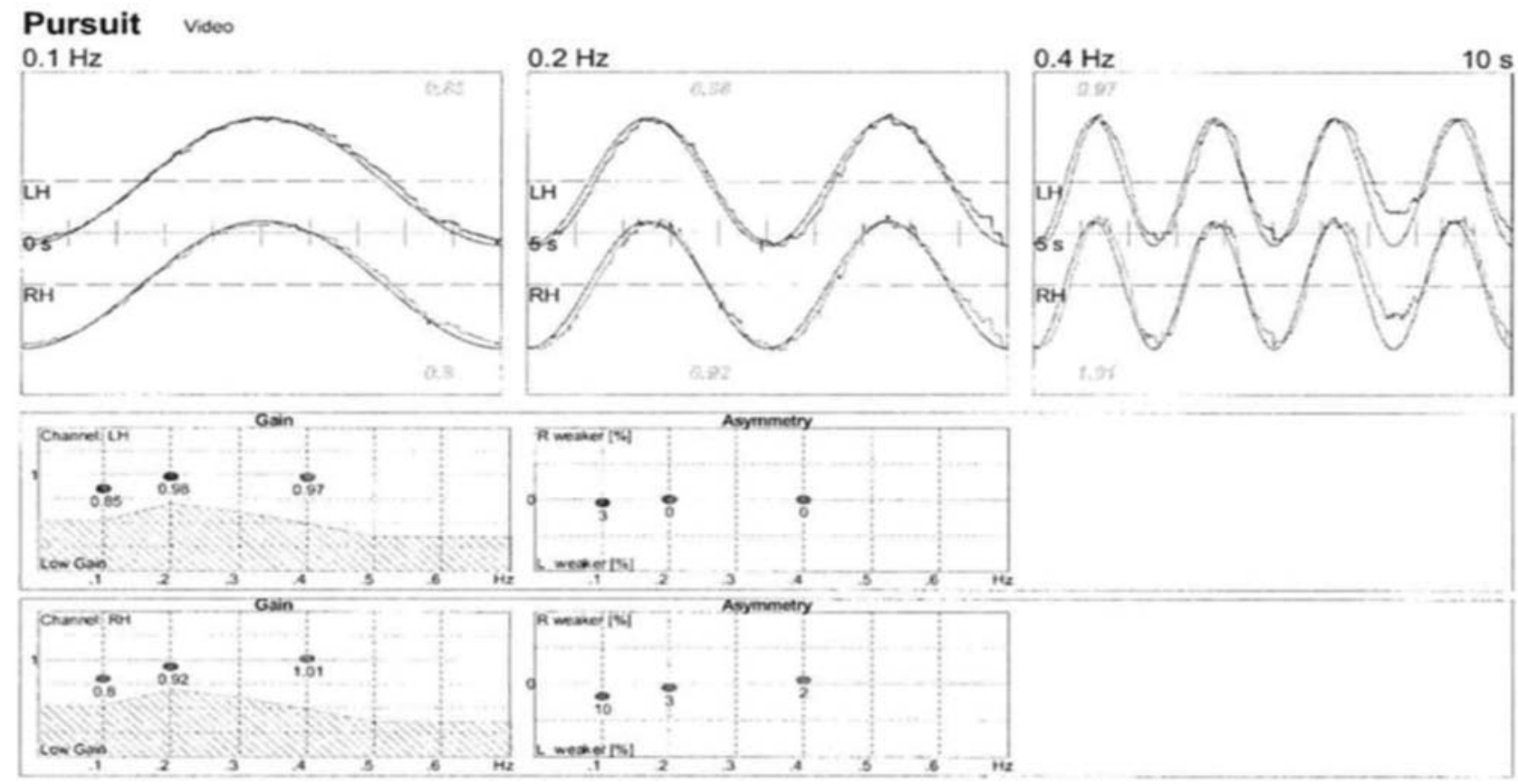

Fig. 3 Smooth pursuit tracing indicates normal gain and phase with subtle saccadic intrusions at $0.4 \mathrm{~Hz}$.

for torsional nystagmus bilaterally. Static positional studies were conducted and a persistent six degree per second right beating nystagmus was observed in head left without fixation, which was suppressed with fixation. Seven degree left beating nystagmus present during head right without fixation, which again was suppressed with fixation. During body left, 11 degree per second right beating nystagmus was observed; and nine degree per second left beating nystagmus was present 


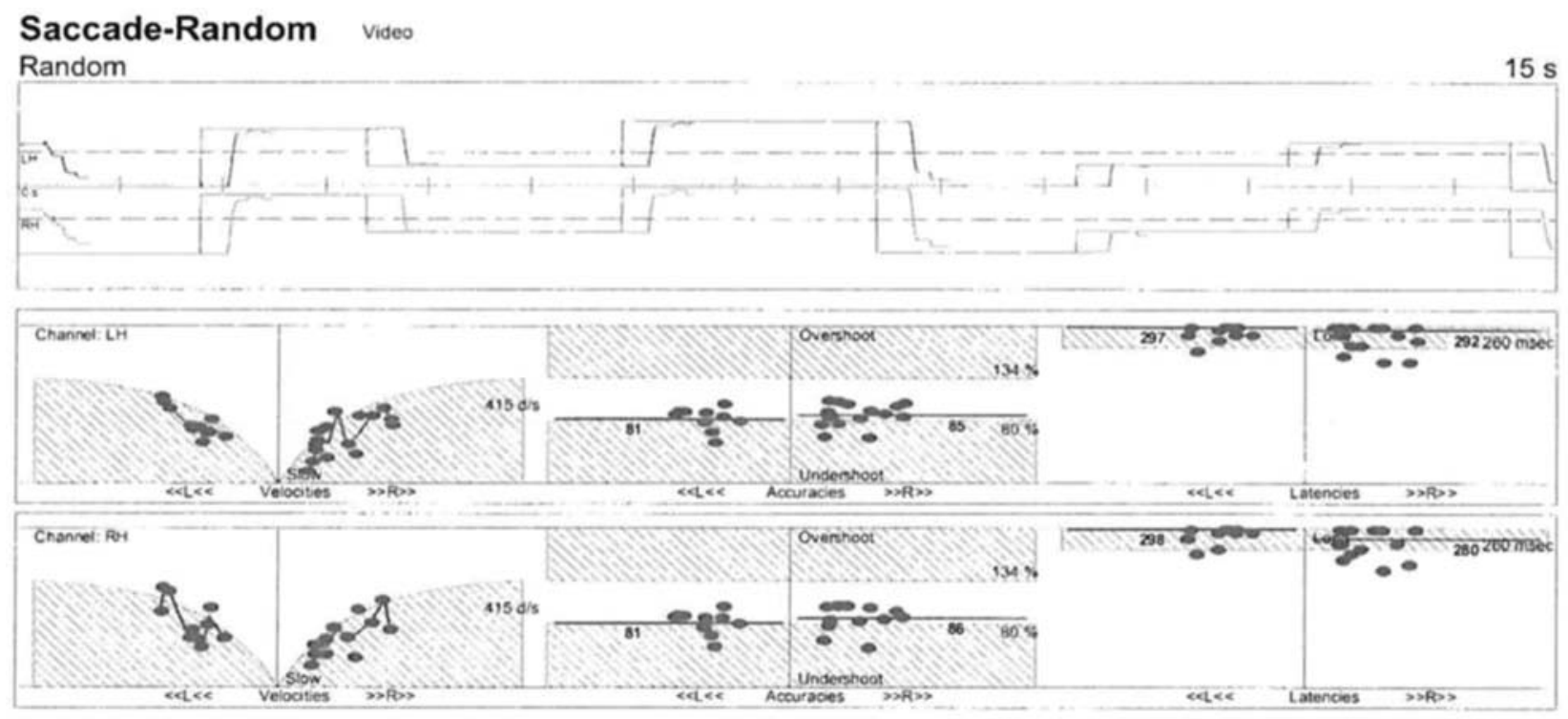

Fig. 4 Saccade results indicate abnormal velocity, consistent undershoots, and abnormally long latencies.

during body right. In both body positions, the nystagmus suppressed with fixation. The patient did not report any feelings of motion during static positional testing. Bilateral bithermal caloric stimulation revealed a significant bilateral weakness. The total slow phase velocity for all irrigations was zero.

\section{Rotational Chair}

SHA testing revealed low gain from 0.01 to $0.64 \mathrm{~Hz}$. Due to low gain measures under 0.2 from 0.01 to $0.32 \mathrm{~Hz}$, symmetry and phase were not calculated at those frequencies (-Fig. 5).

VFX with fixation light revealed normal gain, with no symmetry or phase calculated as gain was below 0.2 , as expected. VVOR with earth-stable optokinetic bars revealed reduced gain with a measurement of 0.5 at $0.16 \mathrm{~Hz}$, which is below normal limits and consistent with previous test results.

Step velocity testing did not produce nystagmus, which resulted in abnormally low gain and time constants in both the clockwise and counterclockwise directions.

Additionally, vHIT performed using the Micromedical System 2000 with vHIT attachment in the lateral plane revealed reduced gain bidirectionally with possible covert saccades
(-Fig. 6). It should be noted that gain was calculated using area-under-the-curve method. The measures of 0.7 fell below clinic normative data and 0.8 were considered borderline. The bulk and weight of the device may have impacted test results.

\section{Discussion}

Collectively, vestibular and balance results indicate severe bilateral horizontal canal paresis with somatosensory dependence. The failure of the angular VOR is most likely contributing to the patient's symptoms of blurred vision and instability while walking in dimly lit and on uneven surfaces. Also unknown is the status of the vertically aligned semicircular canals as vHIT was only performed in the lateral plane. It is important to note that neither cervical nor ocular vestibular evoked myogenic potentials were completed. Therefore, the function of the otolith organs and inferior vestibular nerve cannot be accurately ascertained. However, his general gait instability and poor performance on the SOT may indicate deficits in these areas as well. Additionally, his history of orthopedic injury and total right knee replacement may have

\section{Rotational Chair Summary}
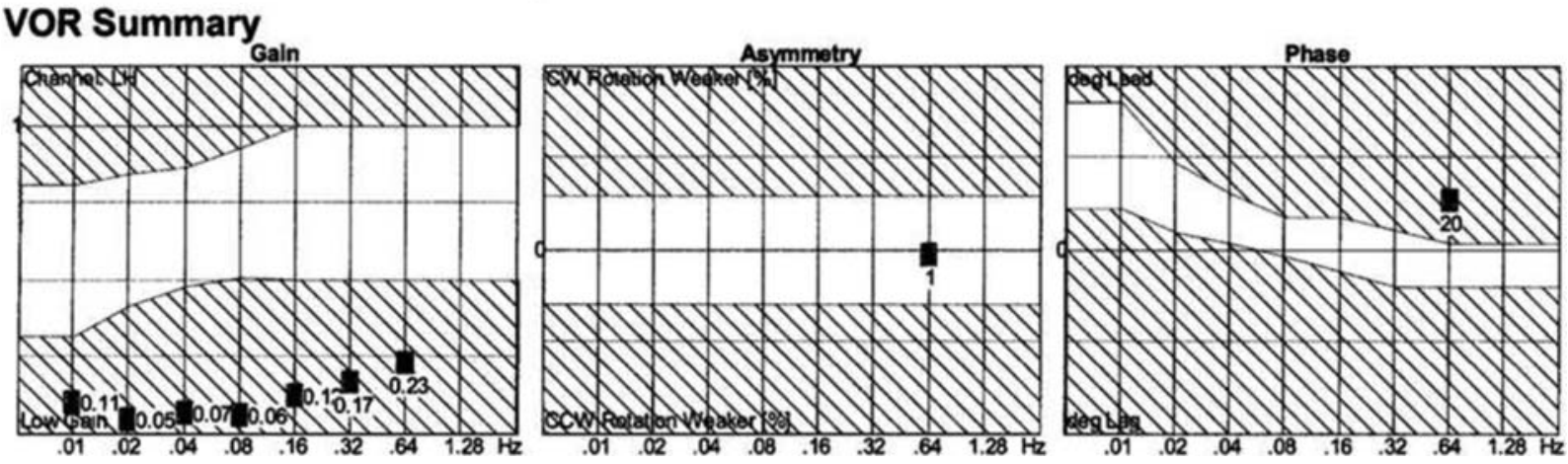

Fig. 5 SHA results indicate severely reduced gain with no interpretable asymmetry or phase measures. SHA, sinusoidal harmonic acceleration. 


\section{Lateral vHIT video}

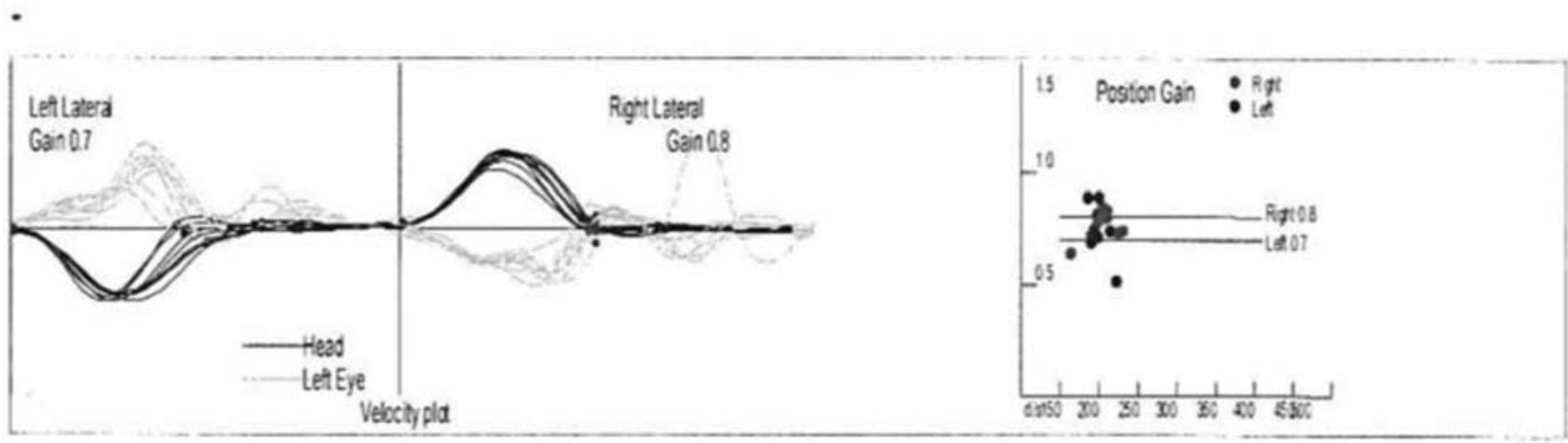

Fig. 6 Lateral vHIT measures indicate abnormal gain, area under the curve with possible covert saccades bidirectionally. vHIT, video head impulse test.

impacted the MCT portion of the test and may explain some of his reported tendency to veer to the right while walking. The absence of results in this patient's caloric testing suggests the presence of either a bilateral dysfunction of the lateral semicircular canals and their afferent pathways, a centrally located lesion or both. Additionally, abnormal saccade parameters (velocity, accuracy, and latency), reduced optokinetic gain, and poor qualitative performance during pursuit evaluations suggest involvement of central system structures. ${ }^{7}$ Also of interest is the presence of nonsymptomatic persistent apogeotropic nystagmus in head/body right and left without fixation. Apogeotropic nystagmus has been reported in individuals with ischemic changes. ${ }^{8}$ In a recent systematic literature review, Macdonald et $\mathrm{al}^{9}$ reported a lack of consistent reporting of the various types of positional nystagmus and their underlying causes. In this review, several of the included studies reported positional horizontal nystagmus, including apogeotropic, in those with a variety of central system site of lesions. Interestingly $85.4 \%$ of subjects in the studies that reported ocular motor testing had abnormalities including abnormal saccades, smooth pursuit, and/or gaze-evoked nystagmus.

While it is possible that CM and ITP are not contributing to the patient's symptoms and vestibular dysfunction, there is some evidence to support their inclusion in the discussion. Lucieer et $\mathrm{al}^{10}$ found that a large percentage of bilateral vestibular hypofunction are idiopathic in nature. A retrospective case study of patients presenting with bilateral vestibular hypofunction found that nearly $10 \%$ of "idiopathic" cases could be attributed to autoimmune disorders, including Susac's disease and Wegener's disease. Although the prevalence of chronic ITP in adults is low, it is reasonable to suspect that this autoimmune disease may be contributing in part to our patient's presentation of bilateral vestibular hypofunction.

Lucieer et al $^{10}$ further identified vascular changes, such as $\mathrm{CM}$, as a definite cause for several cases of bilateral vestibular hypofunction. CM causes changes in the brain vasculature which can result in white matter lesions, lacunar infarcts, and microbleeds. These vascular changes in turn have been noted to impact patient function. As outlined in a retrospective study by Okroglic et $\mathrm{al}^{2}{ }^{2} 27.8 \%$ of patients with documented cases of CM also suffered from gait apraxia. Other commonly co-occurring clinical symptoms included dementia, stroke symptoms, transient ischemic attack symptoms, incontinence, vertigo, and seizures. ${ }^{2}$

Further evidence of this need to diagnose CM as early as possible was shown in a study done by Cerchiai et al, ${ }^{11}$ in which they researched possible underlying causes of dizziness in neurovascular patients. This study identifies CM as "small vessel disease" (SVD). This study examined 60 patients with SVD and categorized them based on neuroimaging results, with either low- or high-burden SVD. After their categorization, the subjects completed vestibular testing, and results were analyzed. Researchers found that there was a higher prevalence of pathological vestibular signs of a peripheral type in patients with lower SVD burden as compared with those with higher burden SVD. Furthermore, similar to our patient, a significant canal paresis was found in 12 patients with low-burden SVD, and in two cases there was evidence of a bilateral vestibular hypofunction. Of note, the two patients identified with a bilateral vestibular hypofunction, denied exposure to any vestibulotoxic agents, and were subsequently referred to neurology for further follow-up. Ultimately, researchers suggested that after a diagnosis of SVD is made, patients should undergo a complete neuro-otologic evaluation due to the high prevalence of peripheral vestibular findings in this population. ${ }^{11}$ There is a paucity of research specifically focused on ITP's effects on the vestibular system, making this case study a unique look into the possible effects of CM and ITP on the vestibular system.

\section{Conclusions}

Information available regarding both CM and ITP suggests both disorders as possible etiologies for this patient's bilateral vestibular hypofunction. ITP is characterized by autoimmune attacks on the body and it is not beyond belief that the vestibular system could be victim of one or more of these attacks. It is also common for ITP to be diagnosed secondary to other disorders such as autoimmune connective tissue disease as well as acute and chronic infections. Currently, there are no other documented cases of patients with bilateral vestibular 
hypofunction and both CM and ITP diagnosis at this time with which we can compare the results of our patient.

With the incidence of $\mathrm{CM}$ and the risk of falling both increasing with age, the importance of vestibular assessment cannot be overstated in this population. Establishing baseline performance and documenting changes within the vasculature and the corresponding changes in vestibular test results may be warranted. Ideally a complete battery of vestibular diagnostic- and function-based tests including VNG, cervical VEMP, ocular VEMP, vHIT (all canals), rotary chair, and CDP should be collected to better define site of lesion in these patients. Ultimately, further research is needed before any concrete statement can be made regarding both CM and ITP's impacts on the vestibular system specifically and global balance function. With regards to management, ITP presents an additional challenge as risk of hemorrhage due to lack of clotting is an unfortunate consequence if platelet levels fall too low. Fortunately for our patient, he has not to date suffered a fall that resulted in severe injury and his platelet levels have stabilized. Proper patient counseling on realistic expectations of postural and balance control with confirmed bilateral hypofunction should be emphasized particularly with those at increased risk of incurring major injury secondary to falls.

Conflict of Interest

None declared.

\section{Acknowledgments}

The authors would like to thank Richard Roberts, PhD, for his review and helpful guidance in preparing this manuscript.

\section{References}

1 de Leeuw FE, de Groot JC, Achten E, et al. Prevalence of cerebral white matter lesions in elderly people: a population based magnetic resonance imaging study. The Rotterdam Scan Study. J Neurol Neurosurg Psychiatry 2001;70(01):9-14

2 Okroglic S, Widmann CN, Urbach H, Scheltens P, Heneka MT. Clinical symptoms and risk factors in cerebral microangiopathy patients. PLoS One 2013;8(02):e53455

3 Bath PM, Wardlaw JM. Pharmacological treatment and prevention of cerebral small vessel disease: a review of potential interventions. Int J Stroke 2015;10(04):469-478

4 Feudjo-Tepie MA, Robinson NJ, Bennett D. Prevalence of diagnosed chronic immune thrombocytopenic purpura in the US: analysis of a large US claim database: a rebuttal. J Thromb Haemost 2008;6(04): 711-712, author reply 713

5 Deng G, Yu S, Li Q et al. Investigation of platelet apoptosis in adult patients with chronic immune thrombocytopenia. Hematology 2017;22(03):155-161

6 Zimmer J, Andrès E, Noel E, Koumarianou A, Blicklé JF, Maloisel F. Current management of adult idiopathic thrombocytopenic purpura in practice: a cohort study of 201 patients from a single center. Clin Lab Haematol 2004;26(02):137-142

7 Leigh R, Zee D. The Neurology of Eye Movements. 4th ed. New York, NY: Oxford University Press; 2006

8 Roberts RA. Technique and interpretation of positional testing. In: Jacobson GP, Shepard NT, eds. Balance Function Assessment and Management. 2nd ed. San Diego, CA: Plural Publishing; 2016

9 Macdonald NK, Kaski D, Saman Y, Al-Shaikh Sulaiman A, Anwer A, Bamiou D-E. Central positional nystagmus: a systematic literature review. Front Neurol 2017;8(April):141

10 Lucieer F, Vonk P, Guinand N, Stokroos R, Kingma H, van de Berg R. Bilateral vestibular hypofunction: insights in etiologies, clinical subtypes, and diagnostics. Front Neurol 2016;7:26

11 Cerchiai N, Mancuso M, Navari E, Giannini N, Casani AP. Aging with cerebral small vessel disease and dizziness: the importance of undiagnosed peripheral vestibular disorders. Front Neurol 2017;8:241 